\title{
An unbiased deep search for small-area molecular structures
}

\author{
A. Heithausen
}

Institut für Physik und ihre Didaktik, Universität zu Köln, Gronewaldstraße 2, 50931 Köln, Germany

e-mail: aheithau@uni-koeln.de

Received 4 January 2006 / Accepted 26 January 2006

\begin{abstract}
Context. Small-area molecular structures (SAMS) resembling clumpuscules proposed as candidates for baryonic dark matter, have been detected in an area where the shielding is too low for them to survive for a long time.

Aims. I present the results of an unbiased deep search for molecular clumpuscules to study the frequency of occurence of such structures. Methods. The area surrounding these structures has been surveyed using the FCRAO $14 \mathrm{~m}$ telescope in the CO $(1 \rightarrow 0)$ transition. The field covered is $20^{\prime}$ by $20^{\prime}$. The resulting rms of the data is only $0.04 \mathrm{~K}$ in a $0.127 \mathrm{~km} \mathrm{~s}^{-1}$ wide channel. Additionally, high-angular resolution observations of the ${ }^{13} \mathrm{CO}$ and $\mathrm{C}^{18} \mathrm{O}(J=1 \rightarrow 0)$ transitions were obtained with the IRAM Plateau-de-Bure Interferometer.

Results. 3 new SAMS have been detected. The structures have very low intensities which makes it impossible to detect them in large scale CO surveys conducted to map the molecular gas of the Milky Way. Their radial velocity is similar to that of the surrounding HI gas. The clouds follow the same size-linewidth relation as found for giant molecular clouds or Galactic cirrus clouds. The observations clearly show that most of the large linewidths observed at low angular resolution are caused by a large velocity difference between the clumps seen at highest angular resolution.

The non-detection of the structures in the high-angular resolution observations of the ${ }^{13} \mathrm{CO}$ and $\mathrm{C}^{18} \mathrm{O}(J=1 \rightarrow 0)$ transitions shows that the ${ }^{12} \mathrm{CO}(J=1 \rightarrow 0)$ transition must have a low optical depth. At an adopted distance of $100 \mathrm{pc}$ the structures have masses of only Jupiter mass or below.

Conclusions. The new observations show that SAMS might be an abundant phenomenon in the interstellar medium but not recognized as such due to their small size. If they are made of ordinary interstellar matter with solar metallicity they likely contribute only little to the total interstellar mass.
\end{abstract}

Key words. dark matter - ISM: abundances - ISM: clouds - ISM: molecules

\section{Introduction}

It has been proposed that most of the baryonic dark matter in the Galaxy is made of small, dense clumps of molecular hydrogen (Pfenniger \& Combes 1994; de Paolis et al. 1995; Gerhard \& Silk 1996; Walker \& Wardle 1998). The detection of $\mathrm{H}_{2}$ is notoriously difficult due to the missing emission lines of that molecule at temperatures below a few hundred K. Sensitive absorption line studies towards many lines of sight have shown that $\mathrm{H}_{2}$ is ubiquitous in our Galaxy (e.g. Shull et al. 2000; Richter et al. 2003a; Jenkins et al. 2003), even in the diffuse medium.

While absorption line measurements indicate that there is significant small-scale structure even in the molecular gas, they do not provide much information on the structure or the form of the absorbing clouds. The recent detection of small-area molecular structures (SAMS) in emission of the CO $(J=1 \rightarrow 0)$ transition (Heithausen 2002, hereafter Paper I) thererfore provided the first chance to study their small scale structure in detail. The detection was somewhat puzzling because the clouds were observed in an area where the shielding is too low for them to survive for a longer time.

Follow-up high-angular resolution observations (Heithausen 2004, hereafter Paper II) showed that the structures resembled those clumpuscules predicted by Pfenniger \& Combes (1994) as important candidates for the baryonic dark matter, albeit warmer and probably less massive. These observations also revealed that all the mass seen on larger scales is contained in smaller structures, a few hundred AU large, with no need for a diffuse molecular component.

Here I present the results of a deep search for further smallarea molecular structures in the area where the first ones have been detected. I will show that these structures are not isolated objects but group in small clusters. Furthermore I will discuss high-angular resolution observations of SAMS2 in the ${ }^{13} \mathrm{CO}$ and $\mathrm{C}^{18} \mathrm{O}(J=1 \rightarrow 0)$ transition which provide information on the optical depth in the same transition of the main isotopomer ${ }^{12} \mathrm{CO}$. These data will allow a better estimate of the mass of these objects and thus of their relative importance. 


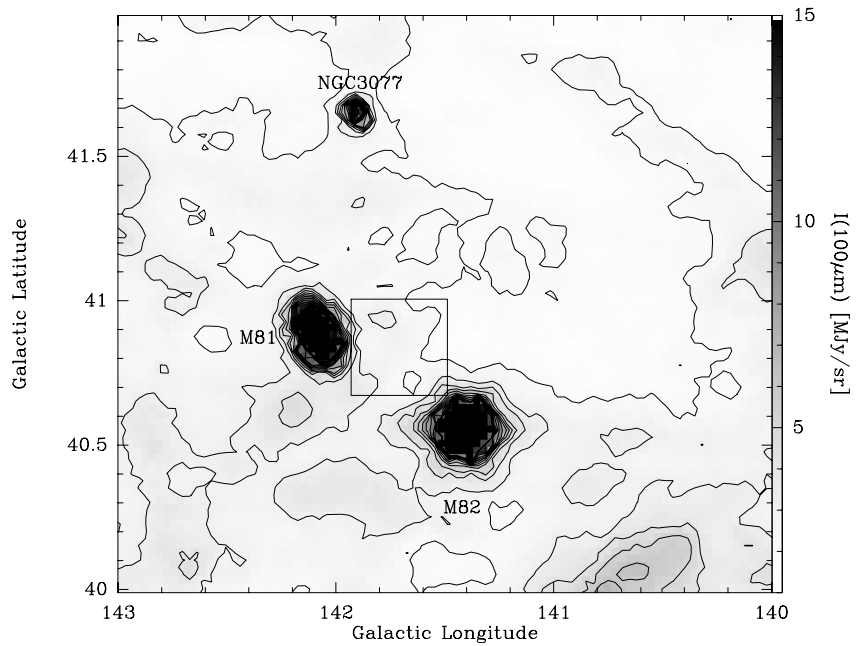

Fig. 1. IRAS $100 \mu \mathrm{m}$ map of the area surrounding the field observed in the $\mathrm{CO}(1 \rightarrow 0)$ line with the FCRAO $14 \mathrm{~m}$ telescope (marked by the square). The intense infrared point sources $\mathrm{M} 81, \mathrm{M} 82$, and NGC 3077 are labelled. Contours are in steps of $1 \mathrm{MJy} \mathrm{sr}^{-1}$ starting at $1 \mathrm{MJy} \mathrm{sr}^{-1}$.

\section{Observations}

\subsection{FCRAO observations}

To study whether SAMS2 is an isolated object, a large-scale map (see Fig. 1) in the $\mathrm{CO} J=1 \rightarrow 0$ transition was obtained with the $14 \mathrm{~m}$ telescope of the Five College Radio Astronomical Observatory (FCRAO) near Amherst in Massachusetts, USA, in April 2003 and January and February 2004. Using the Sequoia 32 pixel receiver, data were taken in the on-thefly mode with an off-position adopted to be free of emission about 1 degree away from the source position. The area was sampled with a spacing of $20^{\prime \prime}$ between individual positions. The angular resolution of the $14 \mathrm{~m}$ telescope at $115 \mathrm{GHz}$ was $44^{\prime \prime}$, the main beam efficiency was 0.45 . The resulting map has a size of $20^{\prime}$ by $20^{\prime}$. The spectra were sampled with a velocity resolution of $0.127 \mathrm{~km} \mathrm{~s}^{-1}$; the resulting rms in the spectra was $0.04 \mathrm{~K}$.

\subsection{Plateau-de-Bure observation}

High angular resolution ${ }^{13} \mathrm{CO}$ and $\mathrm{C}^{18} \mathrm{O}$ data in the $J=1 \rightarrow$ 0 transition were obtained with the Plateau-de-Bure interferometer near Grenoble in the French Alps between May and December 2004. The observations were conducted in the $\mathrm{C}$ and D configuration which gives an angular resolution of $3^{\prime \prime}$ by $3^{\prime \prime}$. The map consists of 6 individual pointings covering an area slightly larger than that observed in the ${ }^{12} \mathrm{CO}(1 \rightarrow 0)$ transition (Paper II). The final spectral resolution was $0.1 \mathrm{~km} \mathrm{~s}^{-1}$ at $110 \mathrm{GHz}$.

\section{Results}

\subsection{CO on larger scales}

Figure 2 shows the results of the unbiased observations of a large area around SAMS2. Maps are presentedintegrated over
$0.2 \mathrm{~km} \mathrm{~s}^{-1}$ wide velocity channels. In these maps four individual clouds can be seen: \#1 corresponds to SAMS2, the other three have not been identified previously. There are even more candidates for molecular clouds in the field but that are however close to the $3 \sigma$ detection limit and thus have to be confirmed. Most of them are found in the velocity range between 5 and $7 \mathrm{~km} \mathrm{~s}^{-1}$ where the other clouds are detected. This velocity range is identical to that of the $\mathrm{HI}$ gas in the same direction.

Note that with the on-off observing method chosen for the observations, signals that do not change much between the on and off position in both velocity and intensity are filtered out. Due to the location of the off-position at $\left(-60^{\prime}, 0^{\prime}\right)$ from the $(0,0)$ position of the map $\left(l_{0}=141^{\circ} .71, b_{0}=40^{\circ} .85\right)$, diffuse clouds larger than 1 degree cannot be detected. Clouds appearing in the on- and off-position with velocities differing by more than the velocity resolution $\left(0.127 \mathrm{~km} \mathrm{~s}^{-1}\right)$ would show up as P-Cygni type line profiles with clouds in the off-position as apparent absorption dips. Such profiles were not detected in the data.

Values from a Gaussian fit to the cloud averaged spectra are listed in Table 1. Line parameters are derived from spectra averaged over the area with significant emission. Radii $r$ given in that table were determined from that area $A$ via $r=\sqrt{A / \pi}$. The center positions were determined from a two dimensional Gaussian fit to the clouds.

\subsection{CO excitation and cloud masses}

In the high-angular resolution ${ }^{13} \mathrm{CO}$ and $\mathrm{C}^{18} \mathrm{O}(J=1 \rightarrow 0)$ observations no signal of more than $0.3 \mathrm{~K}(3 \sigma)$ was detected, compared to lines up to $6 \mathrm{~K}$ in the same velocity range of the ${ }^{12} \mathrm{CO}$ line (Paper II). In typical Galactic molecular clouds both the ${ }^{12} \mathrm{CO}$ and the ${ }^{13} \mathrm{CO}(J=1 \rightarrow 0)$ lines are readily detected with a line ratio of $T\left({ }^{12} \mathrm{CO}\right) / T\left({ }^{13} \mathrm{CO}\right) \approx 3-5$ (e.g. Falgarone et al. 1998), much higher than one would expect from the isotopic ratio $\left[{ }^{12} \mathrm{C}\right] /\left[{ }^{13} \mathrm{C}\right]=66$ (e.g. Bensch et al. 2001) under the assumption that the lines are optically thin. It is therefore concluded that (at least) the ${ }^{12} \mathrm{CO}$ line is optically thick. For SAMS2 the intensity ratio is much higher, $T\left({ }^{12} \mathrm{CO}\right) / T\left({ }^{13} \mathrm{CO}\right) \geq 20$, which means that the ${ }^{12} \mathrm{CO}$ line has a significantly lower optical depth than the same line in other Galactic molecular clouds.

The $\mathrm{H}_{2}$ masses of the structures were therefore determined in two ways:

- adopting a $\mathrm{CO}$ to $\mathrm{H}_{2}$ conversion factor of $X_{\mathrm{CO}}=1.5 \times$ $10^{20} \mathrm{~cm}^{-2}\left(\mathrm{~K} \mathrm{~km} \mathrm{~s}^{-1}\right)^{-1}$, i.e. the Galactic value (Hunter et al. 1997);

- adopting optically thin $\mathrm{CO}$ emission and a $\mathrm{CO}$ abundance of $N_{\mathrm{CO}} / N_{\mathrm{H}_{2}}=1.0 \times 10^{-4}$ (Sutton et al. 1995).

Using the X-factor and adopting a distance of $100 \mathrm{pc}$ to the clouds I derive masses in the range of Jupiter masses $\left(M_{\mathrm{J}}=\right.$ $\left.0.001 M_{\odot}\right)$. The mass for SAMS2 determined in this way is in agreement with that determined from the IRAM $30 \mathrm{~m}$ data (Paper I) given the uncertainties in the determination of the radii and the respective main beam efficiencies of the telescopes used. 


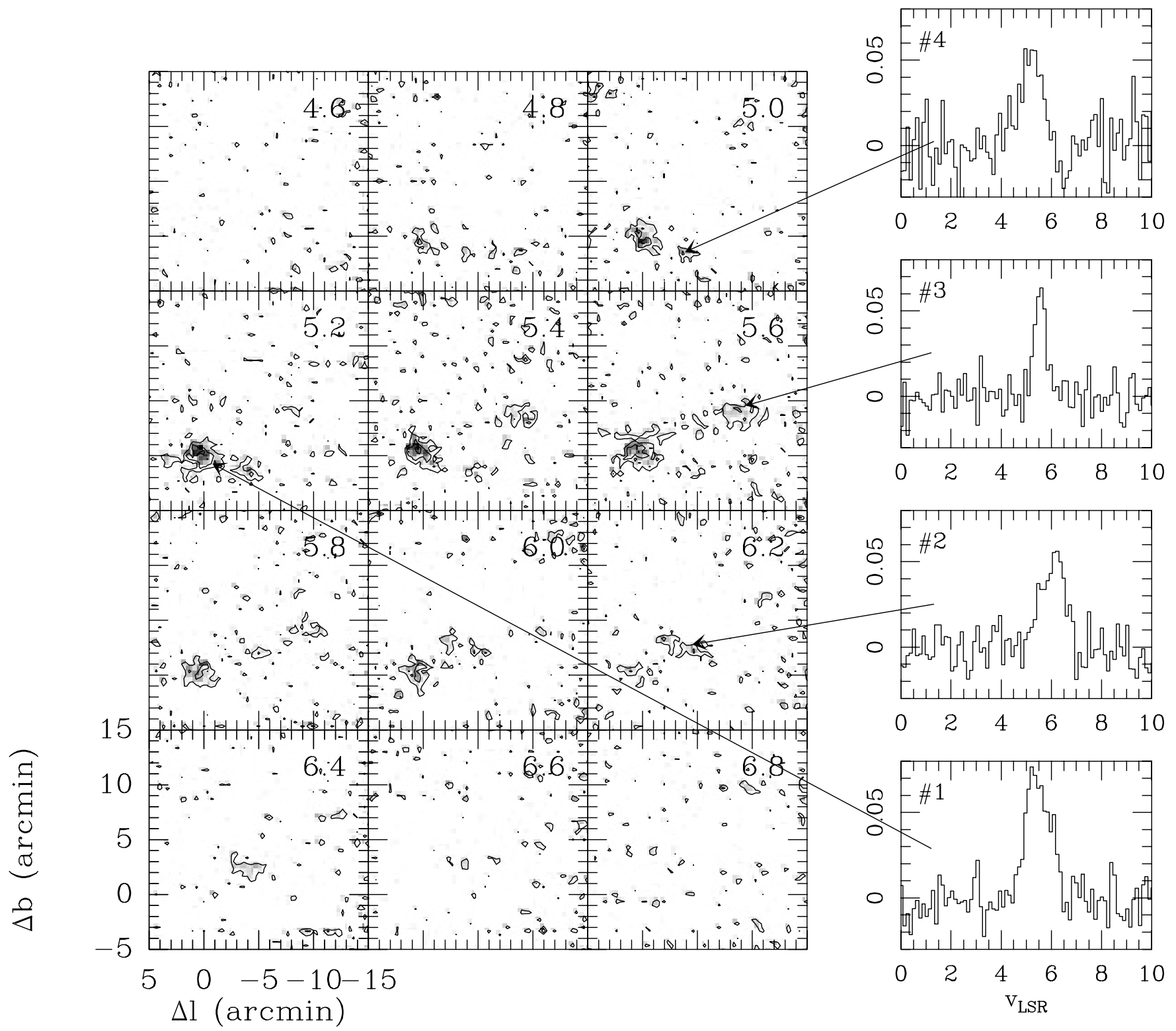

Fig. 2. Channelmaps of the large-scale CO observations obtained with the FCRAO $14 \mathrm{~m}$ telescope. The velocity with respect to the local standard of rest of each channel is indicated in the upper right corner of each map. Offsets in Galactic coordinates are relative to $l_{0}=141^{\circ} .71, b_{0}=40^{\circ} 85$. Contours are every $0.06 \mathrm{~K}$ starting at $0.06 \mathrm{~K}(2 \sigma)$. To the right spectra averaged over the individual substructures are displayed. The temperature scale is $T_{\mathrm{A}}^{*}$ and the velocity $v_{\mathrm{LSR}}$ in $\mathrm{km} \mathrm{s}^{-1}$.

Assuming optically thin emission and adopting an excitation temperature $T_{\mathrm{ex}}=20 \mathrm{~K}$ (see Paper II) results in $\mathrm{H}_{2}$ masses that are a factor of 13.6 lower than those determined with the $\mathrm{X}$-factor. Note that the $\mathrm{CO}$ abundance in regions with low shielding is expected to be lower (e.g. van Dishoeck \& Black 1988) than the value for large molecular clouds given by Sutton et al.; the masses determined in that way are therefore lower limits to the real value (provided the distance is correct).

\section{Discussion}

\subsection{Velocity}

The observations presented in this paper show clearly that SAMS break up in more clumps when going from low to high angular resolution, i.e. SAMS2 seen with the FCRAO telescope shows up as a single structure with an indication of further substructure. Observed with the IRAM $30 \mathrm{~m}$ telescope, at least two substructures are visible (Paper I). At high angular resolution observed with the PdB interferometer, at least 6 smaller stuctures are observed, some of which are even unresolved (Paper II). As the angular resolution increases the line of sight line width decreases from about $1 \mathrm{~km} \mathrm{~s}^{-1}$ at a resolution of $44^{\prime \prime}$ to about $0.4 \mathrm{~km} \mathrm{~s}^{-1}$ at $3^{\prime \prime}$. It is obvious that most of the large line width at low angular resolution is caused by a large velocity gradient among the smallest visible structures.

I therefore first analyse the size-linewidth relation, which provides a good estimate of the amount of turbulence. However before we use the data from Papers I and II to compare them 
Table 1. Parameters for the molecular structures obtained from the FCRAO data.

\begin{tabular}{llllllllll}
\hline \hline Number & $l$ & $b$ & $T_{\mathrm{A}}^{*}$ & $\begin{array}{l}\mathrm{rms} \\
(\mathrm{K})\end{array}$ & $\begin{array}{l}v_{\mathrm{lsr}} \\
\left.(\mathrm{km} \mathrm{s})^{-1}\right)\end{array}$ & $\begin{array}{l}\Delta v \\
\left(\mathrm{~km} \mathrm{~s}^{-1}\right)\end{array}$ & $\begin{array}{l}\operatorname{Radius}^{(1)} \\
\left({ }^{\prime}\right)\end{array}$ & $\begin{array}{l}M_{\mathrm{H}_{2}}^{(2)} \\
10^{-3}\left(\frac{d}{100 \mathrm{pc}}\right)^{2} M_{\odot}\end{array}$ & Identification \\
\hline$\# 1$ & 141.723 & 41.860 & 0.072 & 0.010 & $5.45 \pm 0.04$ & $1.13 \pm 0.08$ & 1.9 & 1.4 & SAMS2 \\
$\# 2$ & 141.666 & 40.895 & 0.053 & 0.011 & $6.07 \pm 0.05$ & $1.07 \pm 0.10$ & 1.6 & 0.7 & - \\
$\# 3$ & 141.518 & 40.922 & 0.060 & 0.012 & $5.57 \pm 0.03$ & $0.52 \pm 0.07$ & 1.5 & 0.3 & - \\
\hline 4 & 141.615 & 40.822 & 0.055 & 0.015 & $5.19 \pm 0.07$ & $1.00 \pm 0.16$ & 0.9 & 0.2 & - \\
\hline
\end{tabular}

Remarks: ${ }^{(1)}$ the radius $r$ is determined from the area $A$ covered by spectra with emission via $r=\sqrt{A / \pi}$. (2) Masses are pure $\mathrm{H}_{2}$ masses not corrected for the contribution of helium; they are calculated using the X-factor. Masses determined assuming a low optical depth are a factor of 13.6 lower (see text).

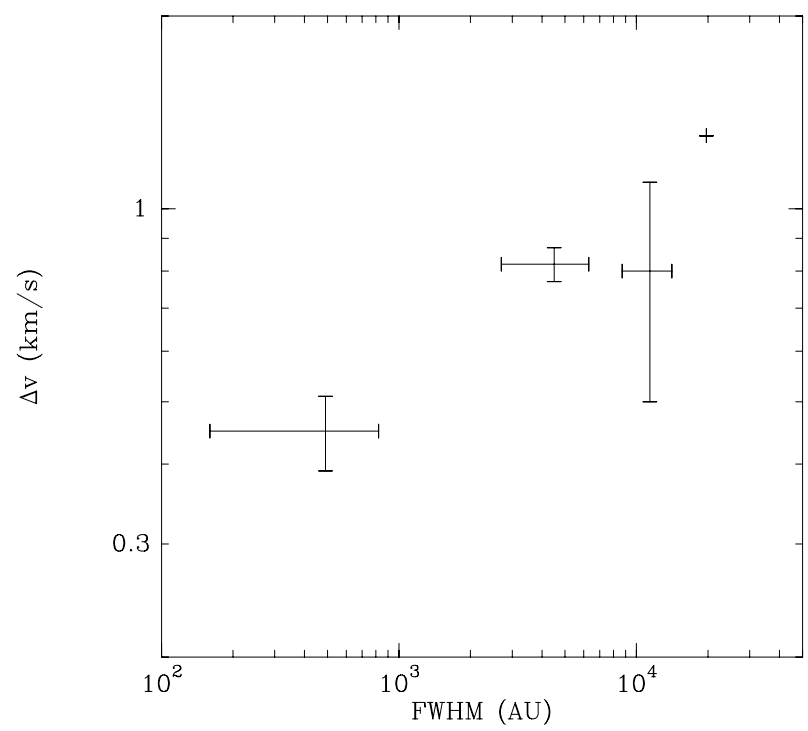

Fig. 3. Size-linewidth relation for SAMS. The correlation includes data also from Papers I and II. Errorbars represent the range of values from the various data sets rather than formal errors.

with the data presented in this paper we have to correct for the different definition of the sizes. In Paper I and II I defined the sizes as full width at half maximum $F W H M$ of the structures derived from a Gaussian fit to the data. In this paper the radius $r$ was derived from the total area covered by significant emission. The latter definition depends therefore on the signal-to-noise ratio $S$ of the data, i.e. a higher $S$ results in a larger size. The correction factor can be calculated from a comparison of their different definitions: $\frac{\sqrt{-2 \ln (S)}}{2.355} F W H M=r$. For the actual $S=$ $1 / 5$ it follows that $r=0.76 F W H M$.

The data corrected in this way are plotted in Fig. 3. This figure also includes a single value for the ensemble of clouds in the area; for that, the radius was calculated from the total area covered by significant emission, and the linewidth from the sum of the spectra shown in Fig. 2. Rather than plotting formal error bars, the figure shows the range of values for the different scales observed. The data closely follow a relation of the form with $\alpha=0.3 \pm 0.1$ which is within the range of values found for giant molecular clouds (Larson 1981) and Galactic cirrus clouds (Heithausen 1996).

\subsection{Abundance}

Even with the deep observations presented in this paper it is hard to determine the relative importance of the small molecular structures. Due to their very small sizes it is clear that we only see those that are very close to us and bright. At a distance three times larger than their actual value, only the structure \#1 (SAMS2) would have been detected with the FCRAO telescope, and only in a single spectrum with very low intensity. So if the distance is indeed $100 \mathrm{pc}$ (see Paper I), we can only see SAMS in a volume of $300 \mathrm{pc}$ radius with the currently available radio telescopes. If SAMS are however a widespread phenomenon they should be easily seen even at larger distances once ALMA comes into operation.

The analysis of the relative importance of SAMS relies furthermore on the assumption that important physical or chemical parameters, such as the $\mathrm{CO}$ abundance or the kinetic temperature, are similar to those found for larger molecular clouds, an assumption which does not necessarily need to be true. As discussed in Sect. 3.2 we possibly overestimate the $\mathrm{CO}$ abundance and thus underestimate the masses and column densities of SAMS. Dirsch et al. $(2003,2005)$ reported the detection of a tiny dust cloud of 4 " diameter seen in projection towards the spiral galaxy NGC 3269. They derive a maximum absorption through the cloud of $A_{\mathrm{B}} \approx 1$ mag. If this dust cloud is also associated with molecular gas it could be the first optically detected SAMS. In that case a more reliable value of the column density and mass could be derived that is significantly higher than that estimated for the SAMS in this paper.

Averaged over the whole $20^{\prime}$ by $20^{\prime}$ area the $\mathrm{CO}$ line has an integrated line strength of $W_{\mathrm{CO}}=0.011 \pm 0.002 \mathrm{~K} \mathrm{~km} \mathrm{~s}^{-1}$. The CO clouds thus account for a molecular hydrogen column density of $N\left(\mathrm{H}_{2}\right)=(0.0012-0.017) \times 10^{20} \mathrm{~cm}^{-2}$; here the lower value is calculated adopting optically thin emission and a Galactic $\mathrm{CO}$ abundance and the upper value adopting a Galactic $X_{\mathrm{CO}}$ factor (see Sect. 3.2). The HI column density of the same area already given in Paper I is $N(\mathrm{HI})=$ $2.1 \times 10^{20} \mathrm{~cm}^{-2}$. That means that only $0.06-0.8$ percent of the total gas in that area is in form of molecular gas if the assumptions made are correct. 


\section{Conclusions}

The deep observations presented in this paper have more than doubled the number of known SAMS. As already discussed in Paper I their velocities are all within the same velocity range as the local HI clouds towards that direction of the sky. They therefore probably are a natural constituent of the interstellar medium, however not recognized as such due to their small angular sizes and low intensities. While on the smallest angular scales the molecular gas of SAMS dominates the total amount of interstellar gas in the corresponding velocity range (as shown in Paper II), on larger scales they may form only a minor fraction. This result relies on the assumption that SAMS are made of ordinary interstellar matter. If they are made of pristine material, only slightly enriched with metals, SAMS could be more massive, because we overestimate the $\mathrm{CO}$ abundance significantly (see Sect. 4.2). In this case they could even be virialized, making them more massive, similar to the clumpuscules proposed by Pfenniger \& Combes (1994). Consequently, they would also contribute more mass to the interstellar medium. Only direct observation of $\mathrm{H}_{2}$ lines, e.g. in absorption to distant stars or quasars, could help resolve this issue.

The detection of Galactic $\mathrm{H}_{2}$ in an HI high-velocity cloud led Richter et al. (1999) to conclude that the cloud may be dissolving. $\mathrm{H}_{2}$ clouds have now been seen towards many extragalactic lines of sight (see Richter et al. 2003a,b; Shull et al. 2000). Some of these clouds have similar physical properties, i.e. high densities and small sizes, as the CO clouds dicussed here. The high detection rate suggests that these are not all in a state of dissolution, but rather that there is a steady process of formation and destruction of $\mathrm{H}_{2}$ clouds in the ISM. Because with respect to fractal index and the size-line width relation SAMS are similar to larger molecular clouds, the observations presented here and in the previous papers also suggest that the structural properties of molecular clouds are imprinted at the beginning of their formation process. High angular resolution
HI data, to compare with the CO data, are necessary to shed further light on this issue. Such data are currently being reduced and will be presented in a forthcoming paper.

Acknowledgements. I thank Arancha Castro-Carrizo (IRAM) for her help during the data reduction of the PdB data and Philipp Richter for comments on the manuscript. This paper is based on observations with the Five College Radio Astronomical Observatory (FCRAO) and the IRAM Plateau-de-Bure interferometer. IRAM is supported by INSU/CNRS (France), MPG (Germany), and IGN (Spain).

\section{References}

Bensch, F., Pak, I., Wouterloot, J. G. A., Klapper, G., \& Winnewisser, G. 2001, ApJ, 562, L185

de Paolis, F., Ingrosso, G., Jetzer, P., \& Roncadelli, M. 1995, A\&A, 299, 647

Dirsch, B., Richtler, T., \& Robinson, L. 2003, A\&A, 408, 929

Dirsch, B., Richtler, T., \& Gomez, M. 2005, AJ, 130, 1141

Falgarone, E., Panis, J.-F., Heithausen, A., et al. 1998, A\&A, 331, 669

Gerhard, O., \& Silk, J. 1996, ApJ, 472, 34

Heithausen, A. 1996, A\&A, 314, 251

Heithausen, A. 2002, A\&A, 393, L41 (Paper I)

Heithausen, A. 2004, ApJ, 606, L13 (Paper II)

Hunter, S. D., et al. 1997, ApJ, 481, 205

Jenkins, E. B., Bowen, D. V., Tripp, T. M., et al. 2003, AJ, 125, 2824

Larson, R. B. 1981, MNRAS, 194, 809

Pfenniger, D., \& Combes, F. 1994, A\&A, 285, 94

Richter, P., de Boer, K. S., Widmann, H., et al. 1999, Nature, 402, 386

Richter, P., Sembach, K. R., \& Howk, J. C. 2003a, A\&A, 405, 1013

Richter, P., Wakker, B. P., Savage, B. D., \& Sembach, K. R. 2003b, ApJ, 586, 230

Shull, J. M., Tumlinson, J., Jenkins, E. B., et al. 2000, ApJ, 538, L73

Sutton, E. C., Peng, R., Danchi, W. C., et al. 1995, ApJS, 97, 455

van Dishoeck, E. F., \& Black, J. 1988, ApJ, 334, 771

Walker, M., \& Wardle, M. 1998, ApJ, 498, L125 